\title{
Carbon Nanotubes and Related Structures: Production and Formation
}

Mark H. Rümmeli, Paola Ayala, and Thomas Pichler

\section{1}

\section{Introduction}

In 1996, Harry Kroto, Robert Curl, and Richard Smalley were awarded the Nobel prize in Chemistry for the discovery of a spherical molecule composed entirely of carbon atoms (Figure 1.1) [34]. This nanometer-scale structure was named "fullerene" due to its resemblance to the highly symmetric architectonic geodesic domes designed by the architect Richard Buckminster Fuller. In the 1980s and early 1990 s extensive research on fullerene theory, synthesis, and its characterization was carried out. In 1991 Iijima [24], presented transmission electron microscopy observations of elongated and concentric layered microtubules made of carbon atoms, which, until then, had mostly been considered as filamentous carbon. This propelled the research related to one of the most actively investigated structures of the last century: nowadays called the carbon nanotubes (CNTs). Despite the ongoing polemic of who should be given credit for the discovery of CNTs [46], it is widely recognized that Iijima's work catapulted carbon nanotubes onto the global scientific stage.

Figure 1.1 shows the ideal molecular models of a $\mathrm{C}_{60}$ fullerene and a singlewalled carbon nanotube. To understand the structure of a carbon nanotube it can be first imagined as a rolled up sheet of graphene (see Figure 1.2), which is a planar-hexagonal arrangement of carbon atoms distributed in a honeycomb lattice. From a fundamental point of view and for future applications the most noticeable features of single-walled CNTs are their electronic (semiconducting or metallic), mechanical (Young modulus $\sim 1 \mathrm{TPa}$ ), optical and chemical characteristics. On the other hand, multiwalled nanotubes are a collection of concentric single-walled nanotubes with different diameters, and their properties are very different from their single-walled counterparts. The promise afforded by carbon nanotubes is varied and they often utilizes their unique electrical properties, extraordinary strength, and heat conduction efficiency. Some applications seek to exploit their structural properties. Examples include high tensile strength fibers and fire resistant materials. 

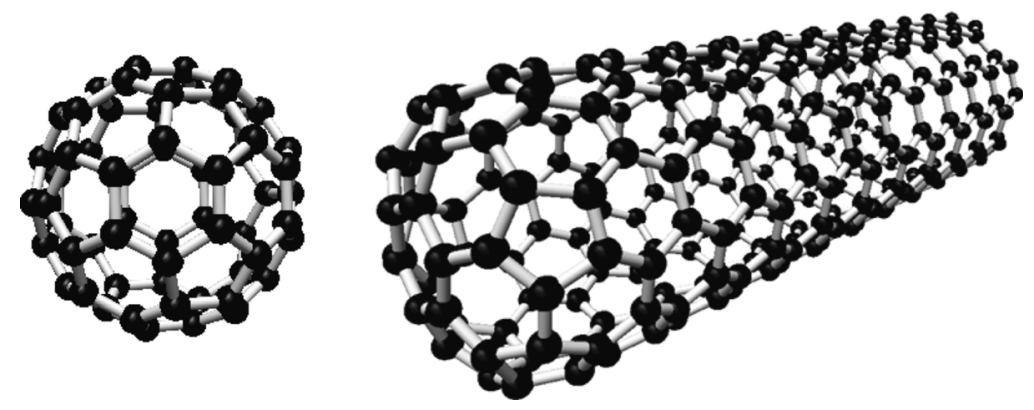

Figure 1.1 Molecular models representing a

structures that can nowadays reach several $\mathrm{C}_{60}$ fullerene (left) and a single-walled carbon micrometers in length but still have very small nanotube caped on one end (right). Fullerenes are closed-cage clusters relatively stable in gas phase, whereas nanotubes are elongated diameters (in the few nanometers order). The end cap of the nanotube can be thought as an incomplete fullerene.

Other applications utilize their electromagnetic properties, for example, artificial muscles [1]. Loudspeakers created from sheets of parallel carbon nanotubes by researchers at the Tsinghua-Foxconn Nanotechnology Research Center in Beijing exemplify their electroacoustic potential. Chemical applications include air and water filtration. Carbon nanotubes are the fastest known oscillators $(>50 \mathrm{GHz})$ and this highlights their incredible mechanical properties. In addition, carbon nanotubes are highly suited as building blocks for molecular electronics. Single-walled CNT, in particular, are attractive due to their electronic structure (semiconducting or metallic)
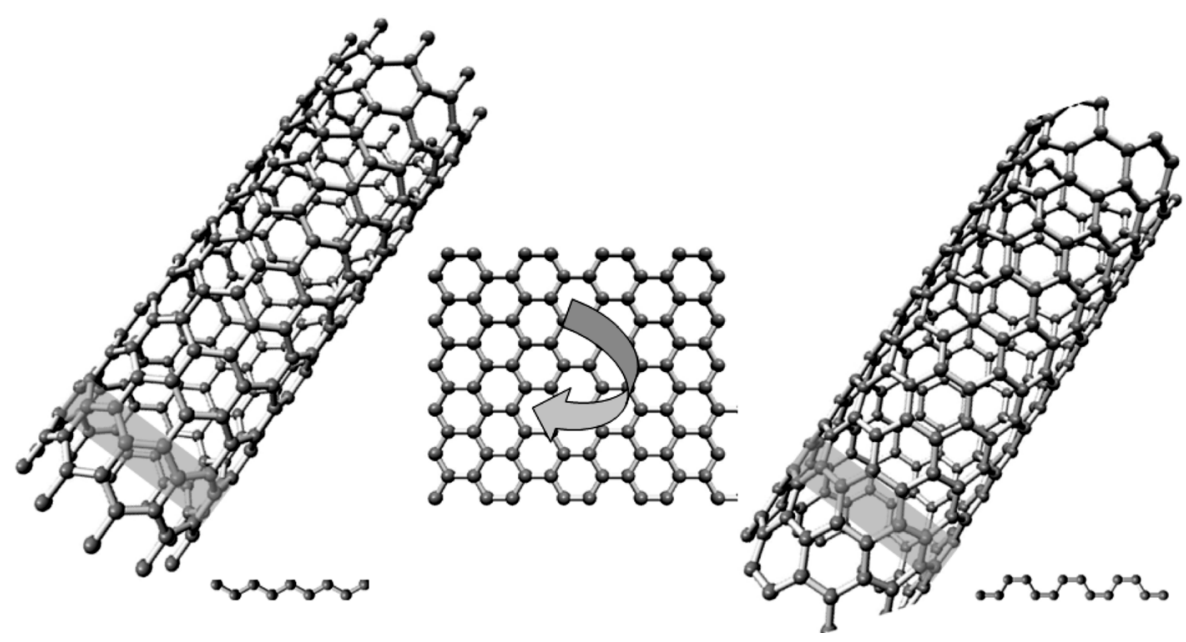

Figure 1.2 A single-walled carbon nanotube can be pictured as a rolled graphene sheet. Nanotubes are classified according to the rolling direction as zigzag, armchair, and chiral.
Multiwalled nanotubes can be pictured as a set of concentric single-walled nanotubes differing from them in their properties. 
being fully described by the tubes diameter and chirality. Field emission transistors (FETs) require semiconducting CNT and high-speed (ballistic) electrical connectors require metallic CNT. However, inherent difficulties in CNT handling pose serious difficulties in their development. For example, the control required to reliably synthesize SWCNT of a given diameter and chirality is still lacking. One of the more promising routes to overcome this difficulty is to functionalize CNT that then allows one to modify their electronic properties in a homogeneous manner. Further, functionalization extends their properties and consequently their application potential. Hence understanding their production and functionalization is crucial for fully exploiting their potential.

There are several techniques for producing single- and multiwalled nanotubes and all of them have advantages as well as disadvantages. In this direction we briefly introduce as examples two of the most established high temperature techniques, namely arc discharge and laser ablation, as well as chemical vapor deposition (CVD) with its most common variants. The literature regarding the synthesis of multiwalled carbon nanotubes is abundant, and in case of the single-walled nanotubes case also it is abundant, but still far from being fully comprehended. For this reason we encourage the reader interested in methods and the specific parameters involved in synthesis processes to deepen in the related review features, chapters in collected editions, books, and journal papers available in the literature $[25,44,60]$. In the following sections, we will rather focus on the contemporary understanding of the growth mechanisms, actual trends, and challenges in carbon nanotube manufacture.

\section{2}

\section{Carbon Nanotube Production}

The state-of-the-art carbon nanotube production encompasses numerous methods and new routes are continuously being developed. The following sections are devoted to give an overall description of the most established nanotube production routes but more detailed information can be found in several review articles.

\subsection{1}

\section{Arc Discharge}

The carbon arc-discharge method is a high temperature process that can be used for the production of nanotubes as well as fullerenes. In fact, mass production of fullerenes was first achieved using arc discharge with the Krätschmer-Huffman method [33]. The derived product and the yields are mainly dependent on the atmosphere and catalysts utilized. This method is probably one of the simplest methods for synthesizing nanotubes on a large scale. However, the simultaneous production of a multimorphology soot demands several purification steps. In the carbon arc-discharge method, an arc is ignited between two graphite electrodes in a gaseous background (usually argon/hydrogen) [11, 23, 24, 64] (Figure 1.3). 


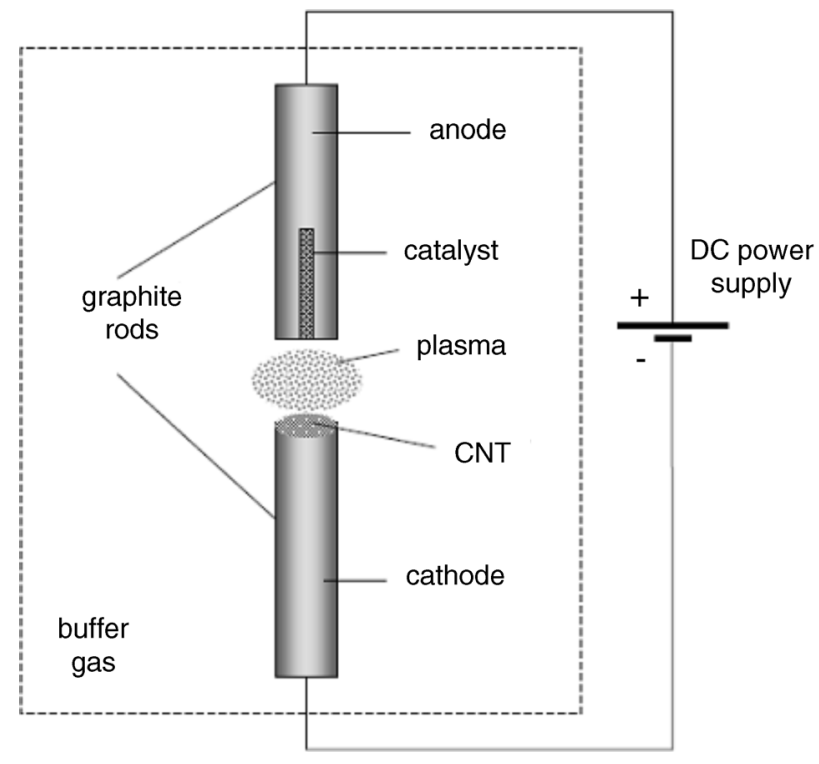

Figure 1.3 Schematic diagram of an arc-discharge system.

The arcing evaporates the carbon and meanwhile it cools and condenses that some of the product forms as filamentous carbon on the cathode. Iijima's work [24] exposed that this filamentous product comprised of multiwalled carbon nanotubes. The optimization of metals being included in the anode led to the growth of single-walled carbon nanotubes [27]. This, in turn, stimulated the successful use of metal catalysts in other techniques for both single-walled and multiwalled carbon nanotube synthesis.

\section{2 .2}

\section{Laser Ablation}

Historically the fullerenes synthesized in the pioneering work of R.F. Curl, H.W. Kroto, and R.E. Smalley were produced using a laser evaporation technique [34]. They directed an intense pulse of laser light on a carbon surface in a stream of helium gas. The evaporated material condenses to yield fullerenes. However it was later noticed that the incorporation of a metal catalyst in the carbon target leads to the formation of SWNT with a narrow diameter distribution and high yields [68]. Further, the SWNT yield and diameter distribution can be varied by controlling the process parameters in the reaction $[3,26,56]$. This technique is not suitable for mass production; however the quality and controlled diameters and diameter distributions offered by laser ablation make them choice SWNT samples for fundamental studies (Figure 1.4). 


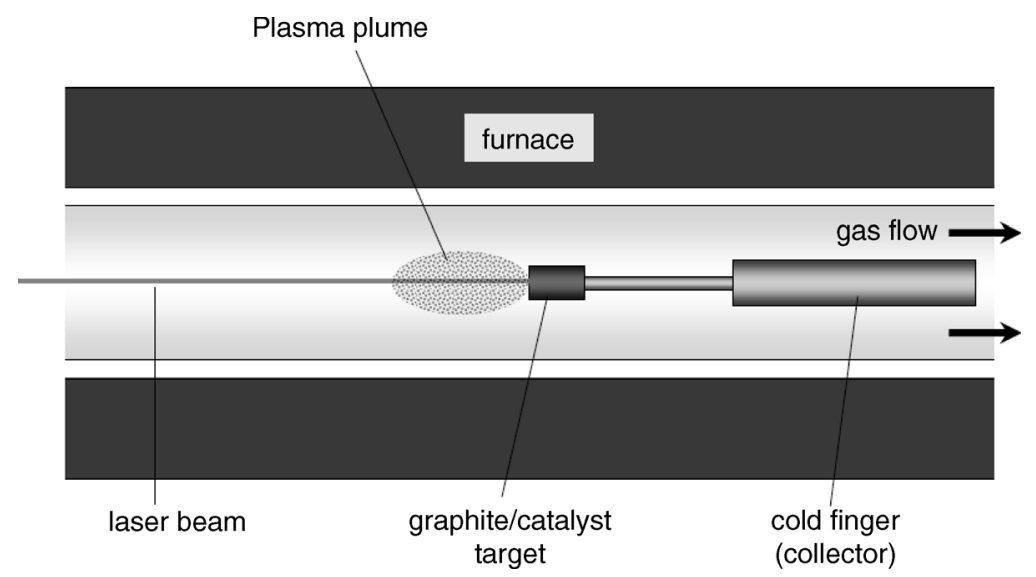

Figure 1.4 Schematics of a laser ablation setup

\section{2 .3}

\section{Chemical Vapor Deposition}

In principle, chemical vapor deposition can be understood as a chemical process in which volatile precursors are used to provide a carbon feed source to a catalyst particle or pore at elevated temperatures $\left(350-1000^{\circ} \mathrm{C}\right)$. The literature available with chemical vapor deposition methods is really extensive, which also shows how this multivariable process can been adjusted in several manners to render various CVD-based methods such as thermochemical CVD (traditional), plasma enhanced CVD, aerosol assisted (AA-CVD), aerogel supported, high pressure CO disproportionation (HiPCO), alcohol catalytic CVD (AACCVD) in ambient or vacuum [5] base pressure, the CoMoCat process, and even a hybrid laser assisted thermal CVD (LCVD) among others. These various forms of CVD in wide use make it the most common route by which CNTs are formed. The relative ease with which one can set up a CVD system also makes of it the most promising route for the mass production of CNTs (Figure 1.5).

In a CVD reaction the catalyst particles can reside in free space, the so-called floating catalysts, or they can reside on a substrate (supported catalysts). In such process, carbon saturates on the catalyst particles and precipitates in the form of a carbon nanotube and is discussed in much detail later (see Figure 1.9). The catalyst particles can also be formed by the decomposition of a volatile precursor as in the HiPCO method that produces relatively larger batches of high quality single-walled carbon nanotubes. This was developed by Nikolaev et al. [48]. Catalysts are formed by the decomposition of a metal containing compound, for example, ferrocene or iron pentacarbonyl in a heated flow of carbon monoxide at pressure between 1 and $10 \mathrm{~atm}$ and temperatures between 800 and $1200^{\circ} \mathrm{C}$. Modification of the process parameters provides control of the SWNT diameter distribution and yield. 


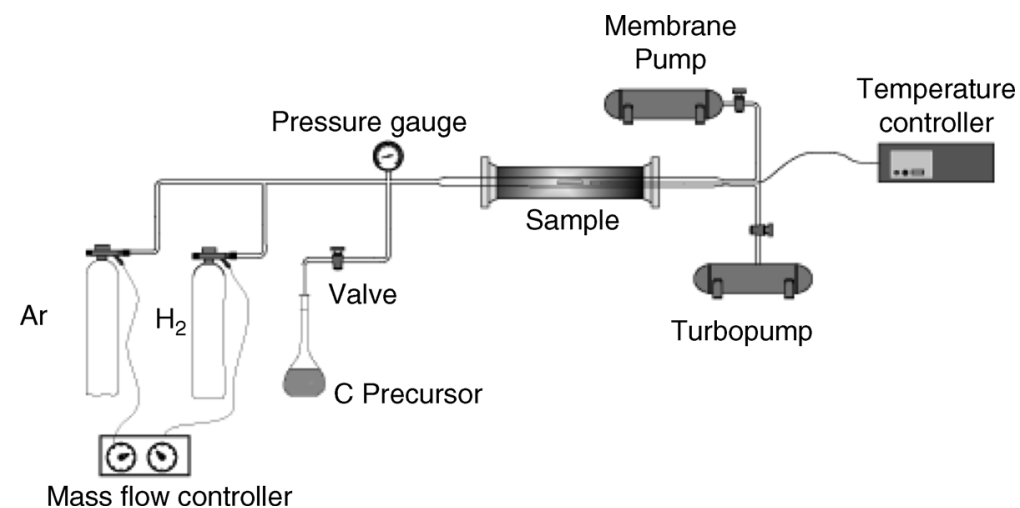

Figure 1.5 Schematic diagram of a chemical vapor deposition system adapted to work in high vacuum conditions, which is only one example of the various CVD-based methods. (Modified from Ref. [5].)

\subsection{4}

\section{Miscellaneous Synthesis Methods}

Apart from the above-mentioned primary synthesis routes there are other variations to these methods and in addition many other synthesis techniques. For example, CNT can be synthesized by dipping hot graphite in cold water [28], ball milling [50], flame synthesis [45], SiC decomposition [35, 37], and graphene scrolling [69] (Figure 1.6).

\section{3}

\section{Catalysts}

The success of catalyst particles in the synthesis of carbon nanotubes is immense. However, it is worth mentioning that carbon nanotubes can be formed without using a catalyst particle [24]. In the case where catalyst particles are used the catalyst particles are considered to play various roles. It is argued the catalyst both nucleates a carbon nanotube and sustains growth. In addition, in CVD, the catalyst is believed to catalytically decompose the carbon feedstock (hydrocarbon).

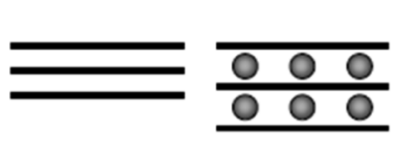

(a)

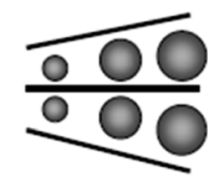

(c)

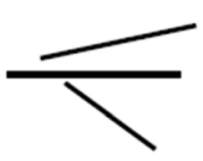

(d)

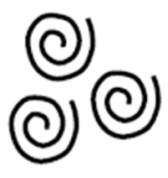

(e)

Figure 1.6 Schematic of the chemical intercalation/exfoliation process. First graphite (a) is intercalated with potassium metal (b) and then exfoliated using ethanol (c) to form a dispersion of carbon sheets (d). A sonication process produces carbon nanoscrolls (e). 


\subsection{1}

\section{Metallic Catalysts}

Historically, the most successful catalysts were transition metals. However, in recent times the successful implementation of nontransition metals and even nonmetals in the synthesis of carbon nanotubes are challenging contemporary concepts in carbon nanotube synthesis.

As a generalization the most commonly used catalysts are $\mathrm{Ni}$, Co, and $\mathrm{Fe}$. These are used independently or mixed with another metal. Ternary catalysts are used often since they usually lead to higher CNT yields. Some examples are, the so-called CoMo Cat CVD synthesis [22], in arc discharge (Rh-Pt) [58] and laser ablation (NiCo) [41]. Rather interestingly though, Fe does not work as a catalyst in laser ablation [38] unless activated with oxygen or hydrogen $[11,38,53]$. In arc discharge it is shown that hydrogen activates Fe [11]. In CVD, Fe is highly effective, however, it should be borne in mind that hydrogen is inherently always present due to the use of decomposing hydrocarbons and often the addition of pure $\mathrm{H}_{2}$ is also implemented. The controversy with iron extends to its oxidation state. This and the roles of hydrogen are discussed later in the enhanced growth section. In terms of using a single metal catalyst for carbon nanotube production only a select few transition metals ( $\mathrm{Ni}, \mathrm{Co}, \mathrm{Pt}$, and $\mathrm{Rh}$ ) are shown to be active in laser ablation [26] As laser ablation conditions are so pure (only pure carbon and the pure metal) it is arguable that only $\mathrm{Ni}, \mathrm{Co}, \mathrm{Pt}$, and $\mathrm{Rh}$ are true catalysts that can catalyze nanotube formation without the assistance of an addcatalyst or enhancer.

Developments over the last few years have demonstrate that many atypical metals, including poor metals can be used for the catalytic growth of carbon nanotubes. Various laser ablation studies showed that atypical metals such as $\mathrm{Cu}$ [54] and poor metals, for example, $\mathrm{Pb}$ and In [53] can be used when activated in the presence of oxygen. In CVD, Takagi et al. developed an activation procedure in which samples preannealed in air yielded SWNT [66]. The technique was used to produce SWNT from $\mathrm{Au}, \mathrm{Ag}$, Pd, and $\mathrm{Cu}$. CVD synthesized SWNT from gold were also synthesized from particles prepared by a block copolymer technique [10]. Gold is generally considered catalytically inactive; however at reduced dimensions it is catalytically active and may have melting points as low as $300^{\circ} \mathrm{C}$. Zang et al. showed that $\mathrm{Pb}$, as found in laser ablation studies, could be used as a catalyst in CVD [74]. Further, they used $\mathrm{Pb}$ as a catalyst for horizontally aligned arrays of SWNT. Yuan et al. synthesized horizontally aligned SWNT from a variety of atypical metal catalysts, including, Mg, $\mathrm{Al}, \mathrm{Cr}, \mathrm{Mo}, \mathrm{Sn}$, and Mn [72].

\subsection{2}

\section{Ceramic Catalysts}

The use of nonmetallic catalysts is of interest in that purification can be easier than for conventional metal catalysts and potentially offers an attractive approach integrating carbon nanotubes with conventional silicon-based technology. The development of nonmetallic catalysts opens up new insights into the growth mechanisms of CNT. 
Most nonmetallic catalysts are ceramics, for example, $\mathrm{SiC}$ and $\mathrm{Al}_{2} \mathrm{O}_{3}$. The first nonmetallic synthesis of carbon nanotubes from a ceramic was shown from the decomposition of $\mathrm{SiC}$ in 1997 [35]. However, the success of transition metal-based catalysts and the shear number of investigators working with CVD left this work largely ignored. However, with recent developments in novel catalysts, including ceramics, and also the ever rising interest in graphene, interest in CNT (and graphene) formation from $\mathrm{SiC}$ and other ceramics is rising. Another ceramic of interest is $\mathrm{Al}_{2} \mathrm{O}_{3}$. Porous alumina is often employed as a template for highly ordered CNT arrays. Usually the catalyst resides at the base of a pore and once subjected to CVD, CNT grow within a pore. However, more recent studies have shown that carbon nanotubes grow from porous alumina without a catalyst particle [6, 59]. This is in agreement with other studies that show many oxides, often used as supports for catalyst or as porous templates for carbon growth, are able to graphitize carbon. The graphene layers apparently stem from step sites [55]. This graphitization potential from alumina is extended to supported catalysis where it is argued that nucleation of a carbon nanotube occurs via the catalysts $(\mathrm{Fe})$ but growth stems from the oxide support rather than the catalyst [57]. Takagi et al. [65] showed the ceramic catalyst concept in its full glory in a study in which they prepared nanosized $\mathrm{Ge}$ and $\mathrm{Si}$ particles on $\mathrm{SiC}$ and nanosized $\mathrm{SiC}$ in $\mathrm{Si}$. In all cases they successfully synthesized SWNT. More recently Huang et al. showed that one can actually grow SWNT from $\mathrm{SiO}_{2}, \mathrm{Al}_{2} \mathrm{O}_{3}$, and $\mathrm{TiO}_{2}$ on supports confirming the work by Rümmeli et al. Often these ceramic techniques are referred to as catalyst free, however, other techniques in which CNT are formed from the vapor phase without a catalytic particle or catalytic support are more aptly described as "catalyst-free" synthesis routes.

\section{3 .3}

\section{Catalyst Free}

The most well known, yet ironically often forgotten, example of catalyst-free synthesis of carbon nanotubes is the work by Iijima [24] that played a key role in the development of carbon nanotube synthesis. In this work MWNT were formed in an arc discharge similar to that used for fullerene synthesis. Another example of "catalyst-free" CNT production was established by using a modified flame route. In this study no catalysts were used and a counter oxygen flow was directed into a methane flame. MWCNT were formed with an oxygen enrichment of 50\% [45]. More recently, MWNT were obtained on a porous carbon surface, at $800^{\circ} \mathrm{C}$ by the decomposition of diluted ethylene [39]. Another catalyst-free route is that in which a hot graphite rod is dipped in cooled water. A sediment is formed that consists of high-purity MWNT [28].

\section{4}

\section{Growth Enhancement}

The use of additives in the synthesis of carbon nanotubes can be used to enhance growth and/or yield. Various additives have been developed with the most notable 
being the use of nominal amounts of $\mathrm{H}_{2} \mathrm{O}$ in supported catalyst CVD. The route Hata developed is commonly known as supergrowth since it yields very long nanotubes with very high purity [18]. The role of $\mathrm{H}_{2} \mathrm{O}$ is argued to be due to the preferential etching of amorphous carbon species [12], water removing undesired surface carbon layers on the catalyst, and are hence, reactivated and the catalytic activity is also dramatically increased [70]. Zhang et al. explored the roles of oxygen and hydrogen [73]. They argue hydrogen has a negative impact that as $\mathrm{H}$ radicals also etch the nanotubes themselves. They suggest that the presence of $\mathrm{O}$ reduces $\mathrm{H}$ radical species via the formation of $\mathrm{OH}$. Alcohol CVD studies by Maruyama et al. suggest $\mathrm{OH}$ removes amorphous carbon [43]. Laser evaporation studies with nominal amounts of water included in the reaction support the notion of amorphous carbon reduction and also suggest oxygen and hydrogen enhance growth and activate the catalysts [12]. Indeed oxygen activation can allow atypical metals to be used as catalysts [53] and hydrogen activates Fe [11, 38]. Sulfur can also be used to enhance CNT formation [3, 9, 38]. The exact nature of the growth enhancement and catalyst activation through additives still remains elusive. However, it is likely that they also play a role in the actual graphitization of carbon species. For example, oxygen-containing gases have been shown to graphitize carbon [49]. In addition, metal oxides are well known to graphitize carbon [55]. However, the oxide state of catalyst particles is not clear. Early work by Baker et al. [7] on filamentous carbon formed in CVD, pointed to the highest activity occurring from the unstable $\mathrm{FeO}$ phase. They argue that this may be due to the defect structure of FeO. More recent in situ XPS studies show conflicting view points; some argue that oxides do not lead to CNT formation (e.g., [19]) while others argue that oxides do play a role (e.g., [42]). The matter is further complicated by in situ TEM data pointing to CNT growth clearly occurring from iron carbide [71]. The issue of growth enhancement and growth are intimately linked and both remain poorly understood. Even so, some important advances in growth have been made and are now briefly discussed.

\section{5}

\section{Growth Mechanisms}

The ever increasing amount of information on CNT growth prevents an exhaustive review in a book chapter like this. Instead the aim here is to focus on major elements in the current understanding of growth, highlight points of controversy, and present new results. In terms of the underlying science carbon nanotube formation may be viewed comprising of two major processes: initial cap formation (nucleation) and circumferential carbon addition (growth).

The formation of an initial cap was first put forward by Dai et al. [14] because the formation of a cap reduces the total surface energy since the basal plane of graphite has a low surface energy. This hemispherical cap formation is referred to as the Yarmulke mechanism. The edges at the base of the cap, it is argued, chemisorb to the metal, hence the existence of energetically costly dangling bonds are avoided. This is supported by calculations by Fan et al. [16] in which they used a series of total energy calculations using density functional theory to show that nucleation 

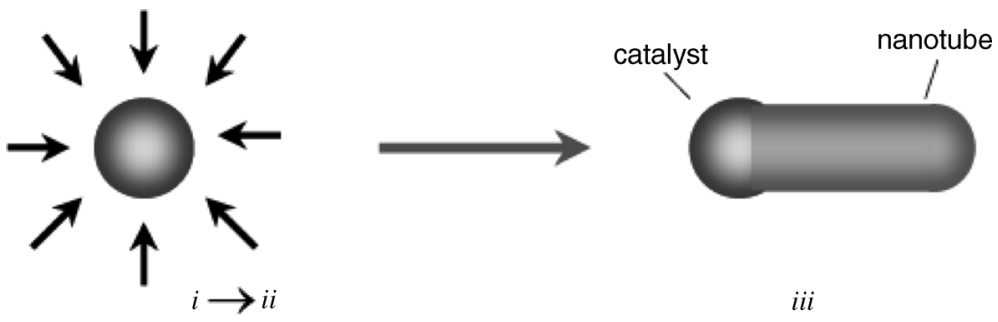

Figure 1.7 VLS schematic: (i, vapor) A semiconductor vapor source adsorbs (black arrows) into the catalyst particle causing it to form a liquid eutectic (ii, liquid). Upon supersaturation a solid precipitates (iii, solid).

of a closed cap or a capped single-walled carbon nanotube is overwhelmingly favored compared to any structure with dangling bond or to a fullerene. Once the hemispherical cap forms, further addition of carbon around the circumference in a cylindrical fashion constitutes the emergence of a tubular structure and is called growth. There are various models proposed for the manner in which the cap formation and addition of carbon to the root of a growing tube occur. However, the vast majority are based around the so-called vapor-solid-liquid (VLS) mechanism [8] to greater or lesser degrees. Hence we briefly introduce the reader to the VLS process before looking at some of the different models proposed for the different catalyst systems. In the VLS process the metal clusters are heated above their metal-carbon eutectic temperature in the presence $\mathrm{C}$. The catalyst particles adsorb carbon, forming a liquid metal-carbide, which eventually consumes the whole catalyst particle. Eventually supersaturation is reached, which leads to the nucleation of solid carbon as the system aims to reach equilibrium. The process is illustrated in Figure 1.7. From this figure it is clear that the role of the catalyst is not only provide a medium through which the nanotube can nucleate and then grow, but also to play an important role in determining the diameter of the tube. Variations to the central VLS model and other suggested models are now briefly discussed according to the manner in which the catalyst particle exists in the reaction; residing in free space (floating catalyst), residing on a substrate (supported catalyst), or no catalyst particle is used (catalyst free).

\subsection{1}

\section{Floating Catalyst Methods}

Synthesis routes that use floating catalysts include arc discharge, laser evaporation, HiPCO, and thermal CVD methods. Common to all these methods is the formation of the catalyst clusters from the vapor phase. In the arc discharge and laser evaporation routes the catalyst material and the carbon are evaporated due to the arc or laser, respectively. In CVD, the decomposition of a metal-based compound (e.g., ferrocene) is the most common way to provide metal catalyst species. The carbon is provided in a similar manner via the decomposition of a hydrocarbon. 
Once the catalyst and carbon species have been vaporized they begin to form clusters as they cool.

As they cool further they coalesce yielding catalyst particles saturated with carbon. As the system cools further the catalyst particles precipitate carbon, forming a nucleation cap and as more carbon is added, a carbon nanotube is extruded. With floating catalyst systems, usually SWNT are obtained. While there is a general agreement that a cap is required for nucleation of a tube, there are disparate ideas as to how this occurs. For example, Gorbunov et al. [17] suggest that precipitating carbon at the particle surface forms graphitic islands which have an initial curvature due to the particle morphology. They argue that the continued addition of carbon will include defects introducing buckling and hence allow curvature until at some stage, a hemispherical cap is arrived at. Further addition of carbon at the cap edges will continue in a cylindrical fashion (tube growth). This mechanism suggests that there is no dependence on the tube diameter and that of the catalyst particle at the point of nucleation. Nasibulin et al. propose that at nucleation the graphitic structure that forms on the surface of the catalyst exceeds that of a Yarmulke-like cap. Here defect forming heptagons provide the required rearrangement for cap lift of (nucleation) and that growth occurs through the constant rearrangement at the root via heptagon formation [47]. This model was developed to explain the larger diameters of the catalyst particles as compared to the obtained nanotubes as observed in postsynthesis TEM studies (Figure 1.8).

However, as pointed out by Dai et al. [14], such studies are complicated because they do not show what the catalyst particle is like at the point of nucleation, for example, ripening process would lead to enlargement of the catalyst particle after cap formation. Dai's findings, on the other hand, suggest there is a direct relationship between the catalyst particle diameter and that of the SWNT. Rümmeli et al. also proposed that the Yarmulke-like nucleation cap is actually dictated by the catalyst particle diameter in the so-called catalyst volume to surface area [56]. They suggested that at the point at which a cooling particle precipitates its carbon, the formation of a Yarmulke cap is not guaranteed. If the particle is too small, insufficient carbon would be available for a stable cap to form and if the particle was too big, the particle would have an excess of carbon that would encapsulate the particle, as also pointed out by Dai et al. [14]. However if the catalyst particle's volume to surface area is just right, a graphitic hemisphere would form providing the nucleation cap (see Figure 1.9). Hence a nucleation window exists. Further carbon addition to the ends would then lead to tube growth. Most theoretical studies tend to point to a strong correlation between the catalyst particle size and the resultant carbon nanotube. Furthermore, theoretical investigations also suggest deviations for very small catalyst particles and larger catalyst particles. For example, molecular dynamics studies show, in general, a strong correlation between the catalyst particle size and the tube diameter at the point of nucleation; however, the studies indicate that for very small catalyst particles (less than $0.5 \mathrm{~nm}$ ), the diameter of the resultant tubes is slightly larger $(0.6-0.7 \mathrm{~nm})$ [15]. Shibuta and Maruyama also conducted molecular dynamics studies to elucidate carbon nanotube nucleation in floating catalyst CVD. They also found a strong correlation between the catalyst 
$12 \mid 1$ Carbon Nanotubes and Related Structures: Production and Formation

(a)
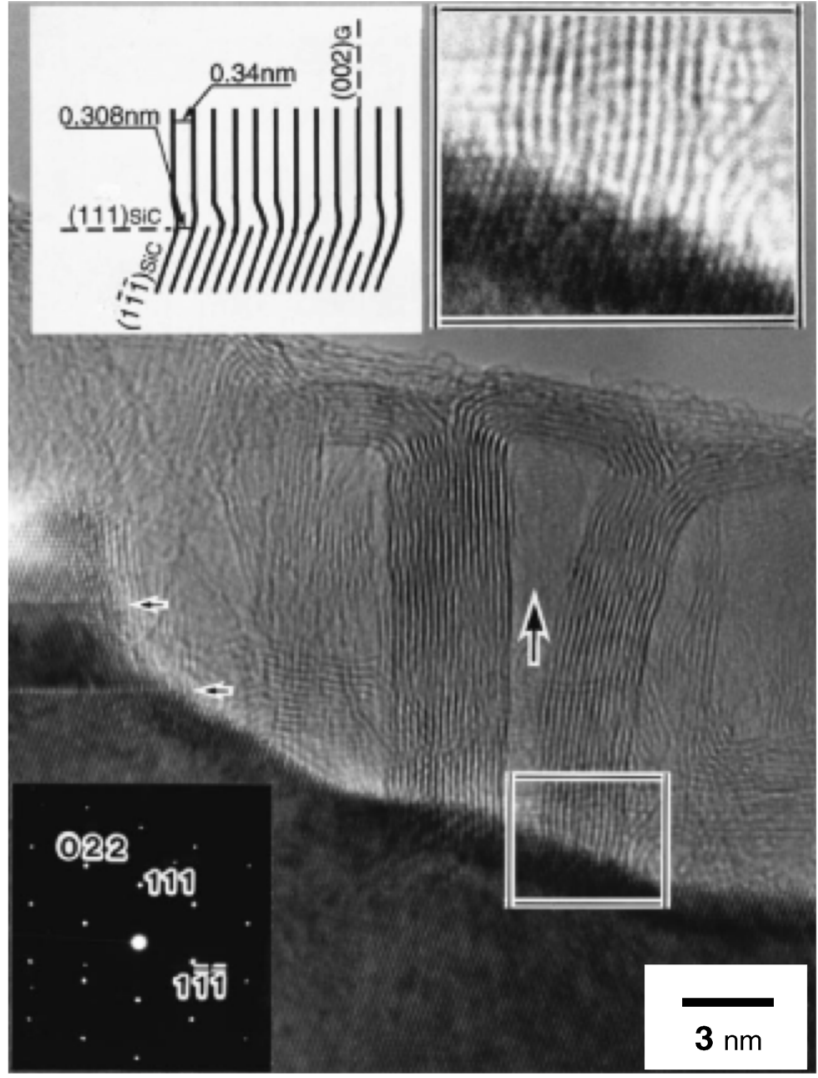

(b)

(111)

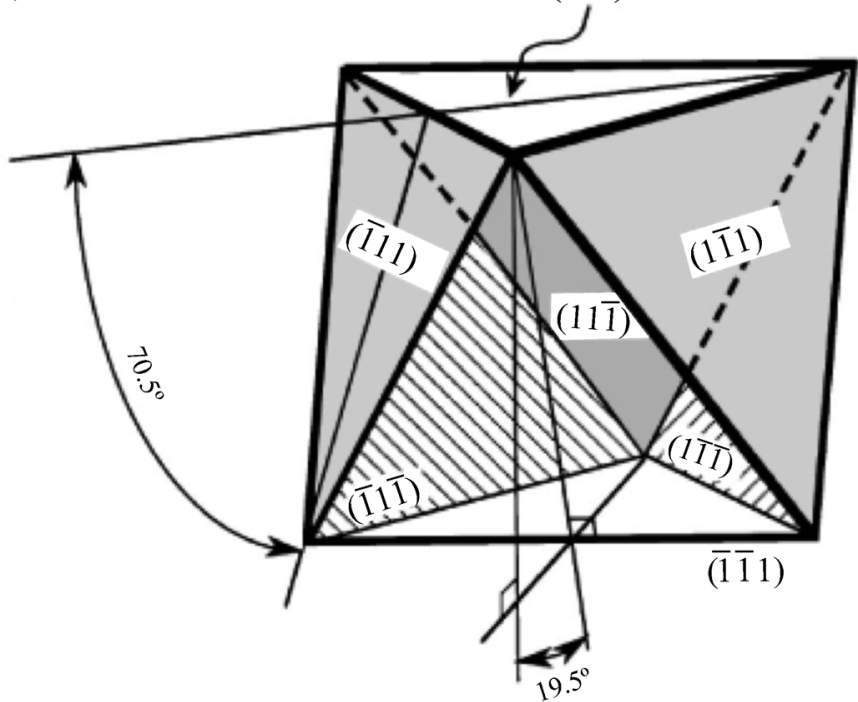

Figure 1.8 (caption see on P. 13) 


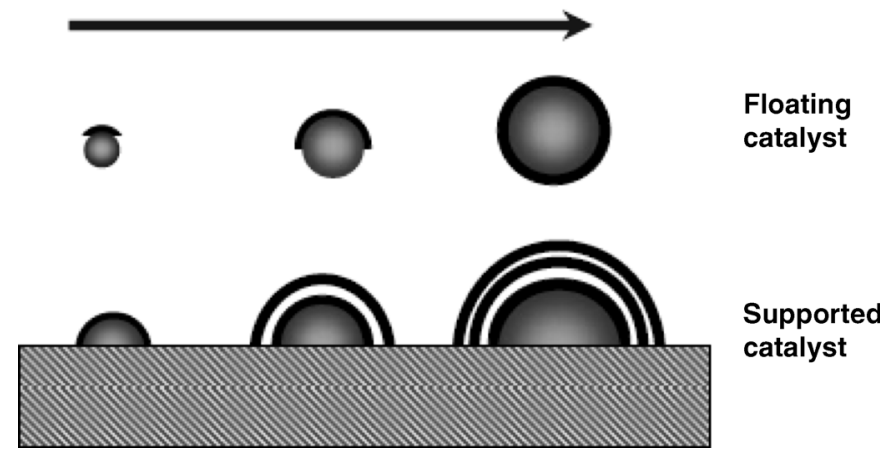

Figure 1.9 Illustration of nucleation variants with floating catalysts (upper) and supported catalysts (lower) under the volume to surface area model. At the point of nucleation, carbon precipitates out of the catalyst particle and in the case of floating catalysts, if insufficient carbon is available then no stable cap is formed, and if too much carbon is available the catalyst particle is encapsulated. Only when the catalyst volume to surface area provides just the right amount of carbon for a stable cap to form, nucleation occur. With supported catalysts since particle encapsulation is prevented by the particle/ support interaction, as the amount of carbon increases (increasing particle size) the number of caps forming also increases.

particle and the nucleation cap (tube diameter). Their work also showed that for larger particles, hump formation on the catalyst particle could lead to cap formations around the hump. In addition their work suggests that very small particle sizes do not lead to tube growth since small caps cannot be lifted as the curvature energy is too high [61]. The various processes are illustrated in Figure 1.10. Hence, molecular dynamics studies point to a nucleation window for SWNT formation with floating catalysts, in agreement with experiment.

\subsection{2}

\section{Supported Catalyst Routes}

In supported catalysis of carbon nanotubes the effect of the substrate on the catalytic process is still unknown since there are many chemical reactions between the substrate and the catalyst metals [22]. However, two modes of growth mechanisms in supported catalysts have been identified: the base growth mode, in which the catalyst particle resides on the support throughout the process, and the tip growth mode, in which the catalyst particle detaches from the support [8]. Indeed, recent advances in transmission electron microscopy have allowed in situ observations of

Figure 1.8 (a) HREM micrograph of the interface between the graphite constructing a carbon nanotube and beta-SiC on the surface of (111) beta-SiC. (b) Schematic of the orientation relationship between one (111) SiC plane, on which carbon nanotubes are standing perpendicularly, and the other [111] SiC planes (Reprinted with permission from Ref. [36]. Copyright 2009, The American Institute of Physics). 
14

1 Carbon Nanotubes and Related Structures: Production and Formation
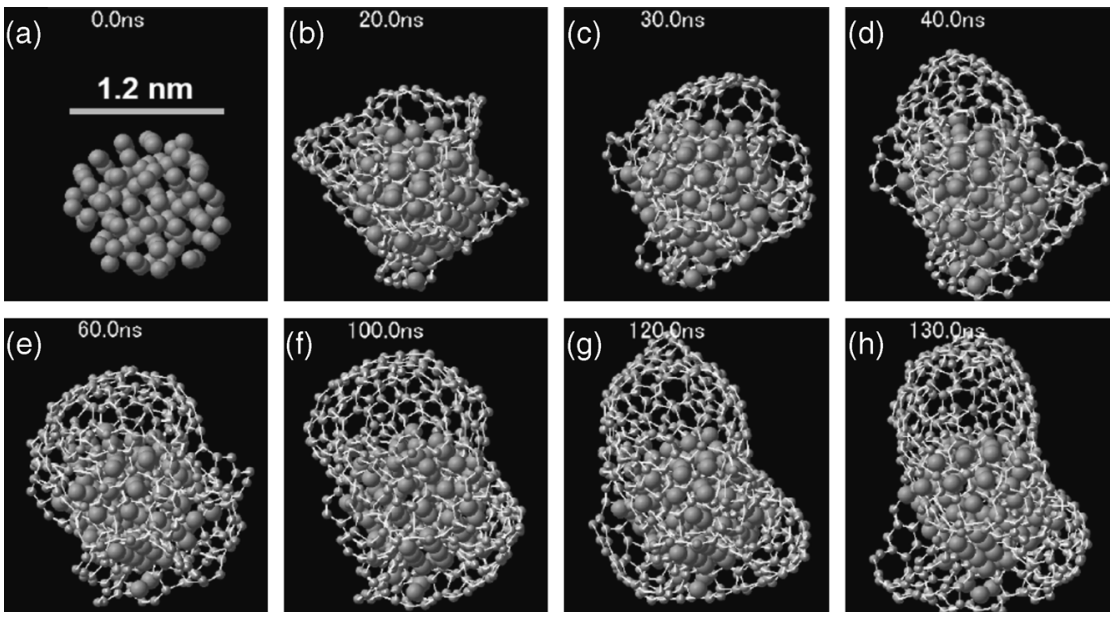

Figure 1.10 Snapshots illustrating the metalcatalyzed nucleation (cap formation) and growth process: a molecular dynamics calculation at $2500 \mathrm{~K}$ for $\mathrm{Ni} 108$ from 0 to $130 \mathrm{~ns}$

(images a-h). Blue spheres represent nickel atoms, and green spheres are carbon atoms. (Images courtesy of Shibuta and Maruyama [63].)

both tip growth [40] and base growth [71]. TEM observations show that for nanotube walls rooted in the catalysts particles, the graphitic walls reside at step sites [21, 52]. They also show that active catalyst particles during growth exhibit liquid-like properties while still retaining solid properties (lattice fringes are clearly observed). This liquid-like behavior does not answer a controversial area for both floating catalysts and supported catalysts, namely, does carbon delivery to a growing graphitic wall occur through bulk diffusion or surface diffusion. Experimental data point to both bulk diffusion [52] and surface diffusion [21] being possible. Likewise, some theoretical investigations suggest that surface diffusion is possible [20]. Other theoretical studies point to bulk diffusion [75]. In terms of specific simulations for catalysts residing on a support, the calculations are more complicated. Shibuta and Maruyama have made preliminary molecular dynamics studies focusing on the effect of the substrate on the metal (catalyst) atoms and have neglected other possible contributions from the substrate in the reaction. Their study show that cap nucleation can occur from catalyst deformation (humps) or from the catalyst particle overall surface similar to their floating catalyst results [62]. A variety of experimental data show a strong correlation between the resultant carbon nanotubes diameter and catalyst particle. In addition, the number of walls increases as the catalyst particles size increases. This is in keeping with the catalyst volume to surface area model described for floating catalysts, but modified to account for the catalyst particle interaction with the support. In this scenario encapsulation of a catalyst particle is prevented by the catalyst/support interaction and results in multiple cap formation as illustrated in Figure 1.9. Regards the root of a carbon nanotube, while most studies assume or show growth to stem from the catalyst particle recent studies by Rümmeli et al. suggest growth may also occur from the 
support itself after nucleation from the catalyst particle has occurred [57]. Such growth is similar to ceramic catalyzed/templated growth. This will be further discussed in the next section.

\subsection{3}

\section{Catalyst-Free Routes}

In this section catalyst free refers to routes in which no catalyst particle is used. However, in some instances it may be that the catalytic activity of the catalyst particle is replaced by some other means. An example is the template synthesis of carbon nanotubes in porous alumina with no catalysts particle present in the pores. In this instance the porous alumina is subjected to CVD conditions similar to catalytic CVD and graphitic walls are found to form in the pores forming MWNT [6, 59]. Similar studies using a variety of oxide nanoparticles found several layers of graphene to form on the surface [55]. Further they appear to root from step sites on the oxide surface. Step sites are well known as catalytic sites. Another route to form graphitic layers or carbon nanotubes is via the decomposition of SiC. This is achieved by annealing $\mathrm{SiC}$ in high vacuum at temperatures between 1400 and $1700^{\circ} \mathrm{C}$. As the $\mathrm{SiC}$ decomposes, graphitic structures like carbon nanotubes emerge. The process is only found to work with trace amounts of oxygen and local EELS studies show oxygen species near and at the surface of the $\mathrm{SiC}[35,37]$. TEM studies show the graphitic layers align with the lattice fringes of the $\mathrm{SiC}$, thus rooting directly into the crystal. This is illustrated in Figure 1.8. The diameter of the CNT and the number of walls formed by the surface decomposition of the $\mathrm{SiC}$ are directly proportional [36]. In addition, the diameter of the nanotubes are determined by the initial cap diameter. The decomposition of the $\mathrm{SiC}$ is argued to occur as

$$
\mathrm{SiC}+\mathrm{CO} \rightarrow \mathrm{SiO}+2 \mathrm{C}
$$

The growth rate of the CNTs is argued to be dependent on the diffusion of $\mathrm{SiO}$ and/ or CO gas through the CNT film. Catalyst-free carbon nanotube formation can also occur without the presence of a ceramic, the most famous being the synthesis of MWNT in the arc discharge without the use of catalysts as first presented by Iijima [24]. A possible argument for their formation is the scroll mechanism in which graphene or graphitic layers roll up. The idea was first proposed in 1960 by Bacon [51] who was studying the filamentous deposit found while arcing two graphite electrodes, which was later shown by Iijima to be MWNT. Theoretical studies [2] show that scrolling is possible. Nucleation is also attributed to the initial formation of a fullerene cap or dome. Recent experiments show such nanoscrolls can also be formed chemically by first exfoliating graphite and then sonicating the sample [69] (see Figure 1.9). The scrolling mechanism is also argued to explain the formation of CNT when hot graphitic rods are dipped in cold water [28]. MWNT have also been synthesized in flame synthesis when nominal amounts of oxygen are included [45]. This route is similar to that used for soot formation, and it is likely that polyaromatic hydrocarbons, PAHs, are involved in their formation. 


\section{6}

\section{Functionalization}

The outer wall of pristine carbon nanotubes is, in principle, conceived as chemically inert. However, this is not always desirable for applications and for this reason, and to further modify the properties of carbon nanotubes in a controlled manner, several functionalization routes have been thought to make them chemically active in the last years. It is worth mentioning that in many cases the word functionalization could be too general to describe the type of changes desired. It can, in principle, mean in-lattice doping, intercalation, molecule or particle adsorption, encapsulation, or even other nonexplored modifications. The several functionalization routes will be discussed in detailed in the following contributions. However, for completeness of this chapter some lines are devoted to the functionalization during synthesis (endohedral doping and sidewall functionalization by heteroatoms).

One typical example for the functionalization of fullerenes was the chemical route for producing $\mathrm{C}_{59} \mathrm{NH}$ [29]. Smith et al. pioneered the endohedral functionalization with molecular structures with the introduction of $\mathrm{C}_{60}$ fullerenes in opened nanotubes, forming the well-known peapods simultaneously [63]. Moreover, endohedral doping of fullerenes was also achieved by the synthesis of different metallofullerenes in the Krätschmer-Huffman furnace [32], and later on, these metallofullerenes were introduced into opened SWCNT by vacuum sublimation in a similar way as Smith et al. had proposed. In this case it is important to notice that this method has also been reported as a unique template reaction in which nanotubes and fullerenes allow the production of metallic nanowires inside SWCNT [31] and MWCNT (Fe filled SWCNT and MWCNT). A second functionalization route can be applied for the formation of heterofullerenes and heteronanotubes by the substitution of carbon by heteroatoms. Boron and nitrogen doped ( $\mathrm{CBx}$ and $\mathrm{CNx}$ ) nanotubes have been successfully incorporated in multiwalled carbon nanotubes by several methods [67]. Although the synthesis of their single-walled counterparts has only recently been reported with successful identification of low doping levels [4, 30].

\section{7}

\section{Purification}

A remaining challenge is the purification of the nanotube material obtained from the different processes. Depending on the synthesis method employed, the yield of undesirable byproducts in the soot varies and these impurities interfere with the properties of the nanotubes. Determining the purity degree of nanotube material is still controversial as no unified standard has been established yet. This is also due to the perspective uses of the synthesized materials. Methods that aim at singlewalled nanotube purification usually employ various oxidation steps, sonication, thermal treatments, chemical acid treatments, among others. However, none of the patented methods is universal. For instance, in a sample free of metal residuals after purification, there still could exist a mixture of nanotubes of different numbers 
of walls. Another clear example could be the case of separated nanotubes. Nowadays it is possible to perform separation of nanotubes with electrophoresis-based as well as ultracentrifugation-based techniques. However, the concept of purity in such a sample is still not fully understood regarding the number of parameters that could be considered. This topic will be discussed more in detail in the later chapters.

\section{8}

\section{Future Perspectives}

The above discussion is intended to summarize the actual research status in the production of carbon nanotubes and to show the line of reasoning regarding growth mechanisms theories. There are several techniques to produce single and multiwalled nanotubes and of course all of them have advantages and disadvantages. The growth mechanisms occurring within these synthesis variants still need to be fully understood and further research is expected in this respect. A notable aspect in this sense is the emergence on nonmetal catalysts and these may well replace metallic catalyst systems due to their compatibility with silicon technology and potential to yield high-purity samples. The literature regarding the synthesis of multiwalled carbon nanotubes is countless and in the single-walled case it is also abundant but still far from being fully comprehended. Great advances have been made in the last few years regarding separation of single-walled carbon nanotubes according to metallicity. However, the purity of those samples and the structure conservation upon the processing methods still needs to be analyzed.

Positive developments have been made in controlling and optimizing the nanotube synthesis, as well as in purification and separation of nanotubes by metallicity and chirality. However, in particular the length separation and the control of the defect concentration and the chirality separation of thick nanotubes still needs to be addressed in order to achieve the final goal of monodisperse samples with full length, chirality, and diameter control.

\section{References}

1 Aliev, A.E., Oh, J., Kozlov, M.E., Kuznetsov, A.A., Fang, S., Fonseca, A.F., Ovalle, R., Lima, M.D., Haque, M.H., Gartstein, Y.N., Zhang, M., Zakhidov, A.A., and Baughman, R.H. (2009) Giantstroke, superelastic carbon nanotube aerogel muscles. Science, 323, 1575.

2 Amelinckx, S., Bernaerts, D., Zhang, X.B., Van Tendeloo, G., and Van Landuyt, J. (1995) A structure model and growth mechanism for multishell carbon nanotubes. Science, 267, 1334.
3 Arepalli, S. (2004) Laser ablation process for single-walled carbon nanotube production. J. Nanosci. Nanotechnol., 4, 317.

4 Ayala, P., Grueneis, A., Gemming, T., Grimm, D., Kramberger, C., Rümmeli, M.H., Freire, F.L., Kuzmany, H., Pfeiffer, R., Barreiro, A., Buechner, B., and Pichler, T. (2007) Tailoring n-doped single and double wall carbon nanotubes from a ondiluted carbon/nitrogen feedstock. J. Phys. Chem. C, 111, 2879. 
5 Ayala, P., Grüneis, A., Kramberger, C., Grimm, D., Engelhard, R., Rümmeli, M.H., Schumann, J., Kaltofen, R., Büchner, B., Schaman, C., Kuzmany, H., Gemming, T., Barreiro, A., and Pichler, T. (2008) Cyclohexane triggers staged growth of pure and vertically aligned single wall carbon nanotubes. Chem. Phys. Lett., 454, 332.

6 Bae, E.J., Choi, W.B., Jeong, K.S., Chu, J.U., Park, G.S., Song, S., and Yoo, Y.U. (2001) Selective growth of carbon nanotubes on pre-patterned porous anodic aluminum oxide. Adv. Mater., 14, 277.

7 Baker, R.T.K., Alonzo, J.R., Dumesic, J.A., and Yates, D.J.C. (1982) Effect of the surface state of iron on filamentous carbon formation. J. Catal., 77, 74.

8 Baker, T. (1982) Formation of filamentous carbon. Chem. Ind. (London), 18, 698.

9 Barreiro, A., Kramberger, C., Rümmeli, M.H., Grüneis, A., Grimm, D., Hampel, S., Gemming, T., Büchner, B., Bachtold, A., and Pichler, T. (2007) Control of the single-wall carbon nanotube mean diameter in sulphur promoted aerosolassisted chemical vapour deposition. Carbon, 45, 55.

10 Bhaviripudi, S., Mile, E., Steiner, S.A., III, Zare, A.T., Dresselhaus, M.S., Belcher, A.M., and Kong, J. (2007) CVD synthesis of single-walled carbon nanotubes from gold nanoparticle catalysts. J. Am. Chem. Soc., 129, 1516.

11 Bystrzejewski, M., Rümmeli, M.H., Lange, H., Huczko, A., Baranowski, P., Gemming, T., and Pichler, T. (2008) Single-walled carbon nanotubes synthesis: a direct comparison of laser ablation and carbon arc routes. J. Nanosci. Nanotechnol., 8, 1.

12 Bystrzejewski, M., Schönfelder, R. Cuniberti, G., Lange, H., Huczko, A., Gemming, T., Pichler, T., Büchner, B., and Rümmeli, M.H. (2008) Exposing multiple roles of $\mathrm{H}_{2} \mathrm{O}$ in high-temperature enhanced carbon nanotube synthesis. Chem. Mater., 20, 6586.

13 Ci, L., Rao, Z., Zhou, Z., Tang, D., Yan, X., Liang, Y., Liu, D., Yuan, H., Zhou, W., Wang, G., Liu, W., and Xie, S. (2002) Double wall carbon nanotubes promoted by sulfur in a floating iron catalyst CVD system. Chem. Phys. Lett., 359, 63.

14 Dai, H., Rinzler, A., Nikolaev, P., Thess, A., Colbert, D.T., and Smalley, R.E. (1996) Single-wall nanotubes produced by metalcatalyzed disproportionation of carbon monoxide. Chem. Phys. Lett., 260, 471.

15 Ding, F., Rose, A., and Bolton, K. (2004) Molecular dynamics study of the catalyst particle size dependence on carbon nanotube growth. J. Chem. Phys., 121, 2775.

16 Fan, X., Buczko, R., Puretzky, A.A., Geohegan, D.B., Howe, J.Y., Pantelides, S.T., and Pennycook, S.J. (2003) Nucleation of single-walled carbon nanotubes. Phys. Rev. Lett., 90, 145501/1.

17 Gorbunov, A., Jost, O., Pompe, W., and Graff, A. (2002) Solid-liquid-solid growth mechanism of single-wall carbon nanotubes. Carbon, 40, 113.

18 Hata, K., Futaba, D.N., Mizuno, K., Namai, T., Yumura, M., and Iijima, S. (2004) Water-assisted highly efficient synthesis of impurity-free single-walled carbon nanotubes. Science, 306, 1362.

19 Hofmann, S., Blume, R., Wirth, C.T., Cantoro, M., Sharma, R., Ducati, C., Hävecker, M., Zafeiratos, S., Schnoerch, P., Oestereich, A., Teschner, D., Albrecht, M., Knop-Gericke, A., Schlögl, R., and Robertson, J. (2009) State of transition metal catalysts during carbon nanotube growth. J. Phys. Chem. C, 113, 1648.

20 Hofmann, S., Csanyi, G., Ferrari, A.C., Payne, M.C., and Robertson, J. (2005) Surface diffusion: the low activation energy path for nanotube growth. Phys. Rev. Lett., 95, 036101.

21 Hofmann, S., Sharma, R., Ducati, C., Du, G., Mattevi, C., Cepek, C., Cantoro, M., Pisana, S., Parvez, A., Cervantes-Sodi, F., Ferrari, A.C., Dunin-Borkowski, R., Lizzit, S., Petaccia, L., Goldoni, A., and Robertson, J. (2007) In situ observations of catalyst dynamics during surface-bound carbon nanotube nucleation. Nano Lett., 7 , 602.

$22 \mathrm{Hu}$, M., Murakami, Y., Ogura, M., Maruyama, S., and Okubo, T. (2004) Morphology and chemical state of Co-Mo catalysts for growth of single-walled 
carbon nanotubes vertically aligned on quartz substrates. J. Catal., 225, 230.

23 Huczko, A., Lange, H., Bystrzejewski, M., Baranowski, P., Ando, Y., Zhao, X., and Inoue, S. (2006) Formation of SWCNTs in arc plasma: effect of graphitization on $\mathrm{Fe}$ doped anode and optical emission studies. J. Nanosci. Nanotechnol., 6, 1.

24 Iijima, S. (1991) Helical microtubules of graphitic carbon. Nature, 354, 56.

25 Jorio, A., Dresselhaus, G., Dresselhaus, M.S. (eds) (2008) Carbon Nanotubes: Advanced Topics in the Synthesis, Structure, Properties and Applications, Topics in Applied Physics, vol. 111, 1st edn, Springer.

26 Jost, O., Gorbunov, A., Liu, X., Pompe, W., and Fink, J. (2004) Single-walled carbon nanotube diameter. J. Nanosci. Nanotechnol., 4, 433.

27 Journet, C., Maser, W.K., Bernier, P., Lamy de la Chapelle, M., Loiseau, A., Lefrant, S., Deniard, P., Lee, R., and Fischer, J.E. (1977) Large-scale production of singlewalled carbon nanotubes by the electricarc technique. Nature, 388, 756.

28 Kang, Z., Wang, E., Gao, L., Lian, S., Jiang, M., $\mathrm{Hu}, \mathrm{C}$. , and $\mathrm{Xu}$, L. (2003) One-step water-assisted synthesis of high-quality carbon nanotubes directly from graphite. J. Am. Chem. Soc., 125, 13652.

29 Keshavarz, M., Gonzalez, K.R., Hicks, R.G., Srdanov, G., Srdanov, V.I., Collins, T.G., Hummelen, J.C., Bellavia-Lund, C., Pavlovich, J., Wudl, F., and Holczer, K. (1996) Synthesis of hydroazafullerene $\mathrm{C}_{59} \mathrm{HN}$, the parent hydroheterofullerene. Nature, 383, 147.

30 Kim, S.Y., Lee, J., Na, C.W., Park, J., Seo, K., and Kim, B. (2005) N-doped doublewalled carbon nanotubes synthesized by chemical vapor deposition. Chem. Phys. Lett., 413, 300.

31 Kitaura, R., Imazu, N., Kobayashi, K., and Shinohara, H. (2008) Fabrication of metal nanowires in carbon nanotubes via versatile nano-template reaction. Nano Lett., 8, 693.

32 Kitaura, R. and Shinohara, H. (2007) Endohedral metallofullerenes and nano-peapods. Jpn. J. Appl. Phys., 46, 881.

33 Krätschmer, W., Lamb, L.D., Fostiropoulos, K., and Huffman (1990)
D.R. $\mathrm{C}_{60}$ : a new form of carbon. Nature, 347, 354.

34 Kroto, H.W., Heath, J.R., O'Brien, S.C., Curl, R.F., and Smalley, R.E. (1985) $\mathrm{C}_{60}$ : buckminsterfullerene. Nature, 318, 14.

35 Kusunoki, M., Rokkaku, M., and Suzuki, T. (1997) Epitaxial carbon nanotube film self-organized by sublimation decomposition of silicon carbide. Appl. Phys. Lett., 71, 2620.

36 Kusunoki, M., Suzuki, T., Honjo, C., Usami, H., and Kato, H. (2007) Closedpacked and well-aligned carbon nanotube films on sic. J. Phys. D, 40, 6278.

37 Kusunoki, M., Suzuki, T., Kaneko, K., and Ito, M. (1999) Formation of self-aligned carbon nanotube by surface decomposition of silicon carbide. Phil. Mag. Lett., 79, 153.

38 Lebedkin, S., Schweiss, P., Renker, B., Malik, S., Hennrich, F., Neumaier, M., Stoermer, C., and Kappes, M.M. (2002) Single-wall carbon nanotubes with diameters approaching $6 \mathrm{~nm}$ obtained by laser vaporization. Carbon, 40, 417.

39 Lin, J.-H., Chen, C.-S., Ma, H.-L., Chang, C.-W., Hsu, C.-Y., and Chen, H.-W. (2008) Self-assembling of multi-walled carbon nanotubes on a porous carbon surface by catalyst-free chemical vapor deposition. Carbon, 46, 1611.

40 Lin, M., Ying Tan, J.P., Boothroyd, C., Loh, K.P., Tok, E.S., and Foo, Y.-L. (2007) Dynamical observation of bamboo-like carbon nanotube growth. Nano Lett., 7, 2234.

41 Lin, X., Rümmeli, M.H., Gemming, T., Pichler, T., Valentin, D., Ruani, G., and Taliani, C. (2007) Single-wall carbon nanotubes prepared with different kinds of NiCo catalysts: Raman and optical spectrum analysis. Carbon, 45, 196.

42 De los Arcos, T., Oelhafen, P., Thommen, V., and Mathys, D. (2007) The influence of catalyst's oxidation degree on carbon nanotube growth as a substrateindependent parameter. J. Phys. Chem. C, 111, 16392.

43 Maruyama, S., Kojima, R., Miyauchi, Y., Chiashi, S., and Kohno, M. (2002) Lowtemperature synthesis of high-purity single-walled carbon nanotubes from alcohol. Chem. Phys. Lett., 360, 229. 
$20 \mid 1$ Carbon Nanotubes and Related Structures: Production and Formation

44 Maser, W., Benito, A.M., Munoz, E., and Teresa Martinez, M. (2008) Carbon Nanotubes: From Fundamental Nanoscale Objects Towards Functional Nanocomposites and Applications, p. 101 Springer, Netherlands.

45 Merchan-Merchan, W., Saveliev, A., Kennedy, L.A., and Fridman, A. (2002) Formation of carbon nanotubes in counter-flow, oxy-methane diffusion flames without catalysts. Chem. Phys. Lett., 354, 20.

46 Monthioux, M. and Kuznetsov, Vladimir L. (2006) Who should be given the credit for the discovery of carbon nanotubes? Carbon, 44, 1621.

47 Nasibulin, A.G., Pikhitsa, P.V., Jiang, H., and Kauppinen, E.I. (2005) Correlation between catalyst particle and single-walled carbon nanotube diameters. Carbon, 43, 2251.

48 Nikolaev, P., Bronikowski, M.J., Bradley, R.K., Rohmund, F., Colbert, D.T., Smith, K.A., and Smalley, R.E. (1999) Gas-phase catalytic growth of single-walled carbon nanotubes from carbon monoxide. Chem. Phys. Lett., 313, 91.

49 Noda, T. and Inagaki, M. (1964) Effect of gas phase on graphitization of carbon. Carbon, 2, 127.

50 Pierard, N., Fonseca, A., Konya, Z., Willems, I., Van Tendeloo, G., and Nagy, J.B. (2001) Production of short carbon nanotubes with open tips by ball milling. Chem. Phys. Lett., 335, 1.

51 Bacon, R. (1960) Growth, structure, and properties of graphite whiskers. J. Appl. Phys., 31, 283.

52 Rodriguez-Manzo, J.A., Terrones, M., Terrones, H., Kroto, H.W., Sun, L., and Banhart, F. (2007) In situ nucleation of carbon nanotubes by the injection of carbon atoms into metal particles. Nat. Nanotechnol., 2, 307.

53 Rümmeli, M.H., Borowiak-Palen, E., Gemming, T., Pichler, T., Knupfer, M., Kalbac, M., Dunsch, L., Jost, O., Silva, S.R.P., Pompe, W., and Büchner, B. (2005) Novel catalysts, room temperature, and the importance of oxygen for the synthesis of single-walled carbon nanotubes. Nano Lett., 5, 1209.
54 Rümmeli, M.H., Güneis, A., Löffler, M., Jost, O., Schönfelder, R., Kramberger, C., Grimm, D., Gemming, T., Barreiro, A., Borowiak-Palen, E., Kalbac, M., Ayala, P., Hübers, H.-W., Büchner, B., and Pichler, T. (2006) Novel catalysts for low temperature synthesis of single wall carbon nanotubes. Phys. Stat. Sol. (B), 243, 3050.

55 Rümmeli, M.H., Kramberger, C., Grüneis, A., Ayala, A., Gemming, T., Büchner, B., and Pichler, T. (2007) On the graphitization nature of oxides for the formation of carbon nanostructures. Chem. Mater., 19, 4105.

56 Rümmeli, M.H., Kramberger, C., Loeffler, M., Jost, O., Bystrzejewski, M., Grueneis, A., Gemming, T., Pompe, W., Buechner, B., and Pichler, T. (2007) Catalyst volume to surface area constraints for nucleating carbon nanotubes. J. Phys. Chem. B, 111, 8234.

57 Rümmeli, M.H., Schäffel, F., Kramberger, C., Gemming, T., Bachmatiuk, A., Kalenczuk, R.J., Rellinghaus, B., Büchner, B., and Pichler, T. (2007) Oxide-driven carbon nanotube growth in supported catalyst CVD. J. Am. Chem. Soc., 129, 15772.

58 Saito, Y., Tani, Y., Miyagawa, N., Mitsushima, K., Kasuya, A., and Nishina, Y. (1998) High yield of single wall carbon nanotubes by arc discharge using $\mathrm{Rh}-\mathrm{Pt}$ mixed catalysts. Chem. Phys. Lett., 294, 593.

59 Schneider, J.J., Maksimova, N.I., Engstler, J., Joshi, R., Schierholz, R., and Feile, R. (2008) Catalyst free growth of a carbon nanotube-alumina composite structure. Inorg. Chim. Acta, 361, 1770.

60 Sgobba, V. and Guldi, D.M. (2009) Carbon nanotubes-electronic/electrochemical properties and application for nanoelectronics and photonics. Chem. Soc. Rev., 38, 165.

61 Shibuta, Y. and Maruyama, S. (2003) Molecular dynamics simulation of formation process of single-walled carbon nanotubes by CCVD method. Chem. Phys. Lett., 382, 381.

62 Shibuta, Y. and Maruyama, S. (2007) A molecular dynamics study of the effect of 
a substrate on catalytic metal clusters in nucleation process of single-walled carbon nanotubes. Chem. Phys. Lett., 437, 218.

63 Smith, B., Monthioux, M., and Luzzi, D. Encapsulated $\mathrm{C}_{60}$ in carbon nanotubes. (1998) Nature, 396, 323.

64 Sun, X., Bao, W., Lv, Y., Deng, J., and Wang, $X$. (2007) Synthesis of high quality singlewalled carbon nanotubes by arc discharge method in large scale. Mater. Lett., 61, 3956.

65 Takagi, D., Hibino, H., Suzuki, S., Kobayashi, Y., and Homma, Y. (2007) Carbon nanotube growth from semiconductor nanoparticles. Nano Lett., 7, 2272.

66 Takagi, D., Homma, Y., Hibino, H., Suzuki, S., and Kobayashi, Y. (2006) Single-walled carbon nanotube growth from highly activated metal nanoparticles. Nano Lett., 6, 2642.

67 Terrones, M., Jorio, A., Endo, M., Rao, A.M., Kim, Y.A., Hayashi, T., Terrones, H., Charlier, J.-C., Dresselhaus, G., and Dresselhaus, M.S. (2004) New direction in nanotube science. Mater. Today, 10, 30-45.

68 Thess, A., Lee, R., Nikolaev, P., Dai, H., Petit, P., Robert, J., Xu, C., Lee, Y.H., Kim, S.G., Rinzler, A.G., Colbert, D.T., Scuseria, G.E., Tománek, D., Fischer, J.E., and Smalley, R.E. (1996) Science, 273, 483.

69 Viculis, L.M., Mack, J.J., and Kaner, R.B. (2009) A chemical route to carbon nanoscrolls. Science, 299, 1361.
70 Yamada, T., Maigne, A., Yudasaka, M., Mizuno, K., Futaba, D.N., Yumura, M., Iijima, S., and Hata, K. (2008) Revealing the secret of water-assisted carbon nanotube synthesis by microscopic observation of the interaction of water on the catalysts. Nano Lett., 8, 4288.

71 Yoshida, H., Takeda, S., Uchiyama, T., Kohno, H., and Homma, Y. (2008) Atomic-scale in situ observation of carbon nanotube growth from solid state iron carbide nanoparticles. Nano Lett., 8, 2082.

72 Yuan, D., Ding, L., Chu, H., Feng, Y., McNicholas, T.P., and Liu, J. (2008) Horizontally aligned single-walled carbon nanotube on quartz from a large variety of metal catalysts. Nano Lett., 8, 2576.

73 Zhang, G., Mann, D., Zhang, L., Javey, A., Li, Y., Yenilmez, E., Wang, Q., McVittie, J.P., Nishi, Y., Gibbons, J., and Dai, H. (2005) Ultra-high-yield growth of vertical single-walled carbon nanotubes: Hidden roles of hydrogen and oxygen. Proc. Natl. Acad. Sci. USA, 102, 16141.

74 Zhang, Y., Zhou, W., Jin, Z., Ding, L., Zhang, Z., Liang, X., and Li, Y. (2008) Direct growth of single-walled carbon nanotubes without metallic residues by using lead as a catalyst. Chem. Mater., 20, 7521.

75 Zhu, Y.-A., Dai, Y.-C., Chen, D., and Yuan, W.-K. (2007) First-principles study of carbon diffusion in bulk nickel during the growth of fishbone-type carbon nanofibers. Carbon, 45, 21. 
